\title{
Lie symmetries of nonlinear boundary value problems
}

\author{
Roman Cherniha ${ }^{\dagger \ddagger}$ and Sergii Kovalenko ${ }^{\dagger}$ \\ † Institute of Mathematics, Ukrainian National Academy of Sciences, \\ 3 Tereshchenkivs'ka Street, Kyiv 01601, Ukraine \\ ¥Department of Mathematics, National University 'Kyiv Mohyla Academy', \\ 2 Skovoroda Street, Kyiv 04070, Ukraine
}

E-mail: cherniha@imath.kiev.ua and kovalenko@imath.kiev.ua

\begin{abstract}
Nonlinear boundary value problems (BVPs) by means of the classical Lie symmetry method are studied. A new definition of Lie invariance for BVPs is proposed by the generalization of existing those on much wider class of BVPs. A class of two-dimensional nonlinear boundary value problems, modeling the process of melting and evaporation of metals, is studied in details. Using the definition proposed, all possible Lie symmetries and the relevant reductions (with physical meaning) to BVPs for ordinary differential equations are constructed. An example how to construct exact solution of the problem with correctly-specified coefficients is presented and compared with the results of numerical simulations published earlier.
\end{abstract}

2010 Mathematics Subject Classification : 22E70, 35K61, 80A22.

\section{Introduction}

It is well known that principle of linear superposition cannot be applied to generate new exact solutions to nonlinear partial differential equations (PDEs). Thus, the classical methods (the Fourier method, the method of the Laplace transformations, and so forth) are not applicable for solving nonlinear PDEs. While there is no existing general theory for integrating nonlinear PDEs, construction of particular exact solutions for these equations is a non-trivial and important problem. Now the most popular methods for construction of exact solutions to nonintegrable nonlinear PDEs are the Lie, Lie-Bäcklund and conditional symmetry methods [1 6 ] Although these methods are very powerful provided the relevant symmetry is known, several other approaches for solving non-integrable nonlinear PDEs were independently suggested during the last decades. Among them the method of compatible differential constraints [7,8, the method of linear invariant subspaces [9], tanh-method and its various modifications [10-14, the method of additional generating conditions [15, 16], and the transformed rational function method [17] should be marked out (see, e.g., Supplements in [18] about other methods).

The Lie symmetries are widely applied to study nonlinear differential equations (including multi-component systems of PDEs) since 60-s of the last century, notably, for constructing their exact solutions. Nevertheless there are a huge number of papers and many excellent books (see, e.g., [15] and papers cited therein) devoted to such applications, one may note that a very small number of them involve Lie symmetries to solve boundary value problems for the given PDEs. To the best of our knowledge, the first papers in this directions were published in the beginning of 1970-s [19] and [20] (the extended versions of these papers are presented in books [21] and [2], respectively). The books, which highlight essential role of Lie symmetries in solving boundary value problems (BVPs) and present several examples, were published only in 1989 [2,22].

The main object of this paper is a nonlinear BVP of Stefan type, which belongs to the class of BVPs with free (moving) boundaries. Boundary value problems of Stefan type are widely 
used in mathematical modeling a huge number of processes, which arise in physics, biology and industry [23 27]. Nevertheless these processes can be very different from formal point of view, they have the common peculiarity, unknown moving boundaries. Movement of unknown boundaries are described by famous Stefan boundary conditions [27,28]. It is well-known that exact solutions of BVPs of Stefan type can be derived only in exceptional cases and the relevant list is rather short at the present time (see [23, 29 35] and papers cited therein).

Nevertheless BVPs with free boundaries are more complicated objects than the standard BVPs with the fixed boundaries, it can be noted that the Lie symmetry method should be more applicable just for solving problems with moving boundaries. In fact, the structure of such boundaries may depend on invariant variable(s) and this gives a possibility to reduce the given BVP to that of lover dimensionality. This is the reason why different authors applied the Lie symmetry method to BVPs with free boundaries ignoring BVPs with fixed boundaries $[19,20,36-38$.

The paper is organized as follows. In Section 2, we discuss the existing definitions of Lie invariance for BVPs and propose their generalization on much wider class of BVPs. As an example the direct application of the definition for the well-known BVP with the fixed boundaries is presented. In Section 3, we apply the definition derived to the class of $(1+1)-$ dimensional BVPs of Stefan type used to describe melting and evaporation of materials in the case when their surface is exposed to a powerful flux of energy [31,39]. The result obtained is an essential generalization of paper [37]. In Section 4, we reduce the problem to BVPs for ordinary differential equations, using Lie symmetry operators obtained in the previous section. An example how to construct exact solution of the problem with correctly-specified coefficients is also presented. Finally, we present conclusions in the last section.

\section{Definition of Lie invariance for BVPs}

We start from a definition of invariance of a BVP under the given infinitesimal operator presented in [2,5] and restrict ourselves on the case when the basic equation of BVP is an $(1+1)$-dimensional evolution PDE of $k$ th-order $(k \geq 2)$. In this case the relevant BVP may be formulated as follows:

$$
\begin{gathered}
u_{t}=F\left(x, u, u_{x}, \ldots, u_{x}^{(k)}\right),(t, x) \in \Omega \subset \mathbb{R}^{2} \\
s_{a}(t, x)=0: B_{a}\left(t, x, u, u_{x}, \ldots, u_{x}^{(k-1)}\right)=0, a=1,2, \ldots, p,
\end{gathered}
$$

where $F$ and $B_{a}$ are smooth functions in the corresponding domains, $\Omega$ and $s_{a}(t, x)$ are a domain with smooth boundaries and smooth curves, respectively. Hereafter the subscripts $t$ and $x$ denote differentiation with respect to these variables, $u_{x}^{(j)}=\frac{\partial^{j} u}{\partial x^{j}}, j=1,2, \ldots, k$. We assume that BVP (11) and (2) has a classical solution (in a usual sense).

Consider the infinitesimal generator

$$
X=\xi^{0}(t, x) \frac{\partial}{\partial t}+\xi^{1}(t, x) \frac{\partial}{\partial x}+\eta(t, x, u) \frac{\partial}{\partial u},
$$

(hereafter $\xi^{0}, \xi^{1}$ and $\eta$ are known smooth functions), which defines a Lie symmetry acting on both $(t, x, u)$-space as well as on its projection to $(t, x)$-space. Let $X^{(k)}$ be the $k$ th-prolongation of the generator $X$ calculated by the well-known prolongation formulae (see, e.g. [1,3]).

Definition 1 [2] The Lie symmetry $X$ of the form (3) is admitted by the boundary value problem (1) and (2) if and only if: 
(a) $X^{(k)}\left(F\left(x, u, u_{x}, \ldots, u_{x}^{(k)}\right)-u_{t}\right)=0$ when $u$ satisfies (11);

(b) $X\left(s_{a}(t, x)\right)=0$ when $s_{a}(t, x)=0, a=1,2, \ldots, p$;

(c) $X^{(k-1)}\left(B_{a}\left(t, x, u, u_{x}, \ldots, u_{x}^{(k-1)}\right)\right)=0$ when $\left.B_{a}\right|_{s_{a}(t, x)=0}=0, a=1,2, \ldots, p$.

The definition can straightforwardly be extended on BVPs for a system of PDEs. However, one easily notes that this definition cannot be applied to BVPs with free boundaries, because such problems contain moving surfaces, say $S_{b}(t, x)=0, b=1, \ldots, q$, where $S_{b}(t, x)$ are unknown functions. Obviously, these functions should be interpreted as additional variables. In [36] (see Appendix 2), a criteria of invariance for BVP with a free boundary was formulated. Another deficiency of Definition 1 appears if one consider BVPs in the unbounded domain $\Omega$ when the boundary conditions for $x=\infty$ arise. In fact, item (b) has no sense in this case and cannot be replaced by the natural passage to the limit, i.e., $x=L, L \rightarrow \infty$. Probably this deficiency for the first time was noted in [40] (see Section 4.3) where the transformation $x=1 / y$ was suggested to avoid the non-regular manifold generated by $x=\infty$.

Now we present a definition which takes into account all possible boundary conditions and is applicable to a wide range of BVPs. Consider a BVP for a system of $n$ evolution equations $(n \geq 2)$ with 2 independent $(t, x)$ and $n$ dependent $u=\left(u_{1}, u_{2}, \ldots, u_{n}\right)$ variables. Let us assume that the $k$ th-order $(k \geq 2)$ basic equations of evolution type

$$
u_{t}^{i}=F^{i}\left(x, u, u_{x}, \ldots, u_{x}^{(k)}\right), i=1, \ldots, n
$$

are defined on a domain $\Omega \subset \mathbb{R}^{2}$ with smooth boundaries. Consider three types of boundary and initial conditions, which can arise in applications:

$$
\begin{gathered}
s_{a}(t, x)=0: B_{a}^{j}\left(t, x, u, u_{x}, \ldots, u_{x}^{\left(k_{a}^{j}\right)}\right)=0, a=1, \ldots, p, j=1, \ldots, n_{a}, \\
S_{b}(t, x)=0: B_{b}^{l}\left(t, x, u, \ldots, u_{x}^{\left(k_{b}^{l}\right)}, S_{b}, \frac{\partial S_{b}}{\partial t}, \frac{\partial S_{b}}{\partial x}\right)=0, b=1, \ldots, q, l=1, \ldots, n_{b},
\end{gathered}
$$

and

$$
\gamma_{c}(t, x)=\infty: \Gamma_{c}^{m}\left(t, x, u, u_{x}, \ldots, u_{x}^{\left(k_{c}^{m}\right)}\right)=0, c=1, \ldots, r, m=1, \ldots, n_{c} .
$$

Here $k_{a}^{j}<k, k_{b}^{l}<k$ and $k_{c}^{m}<k$ are the given numbers, $s_{a}(t, x)$ and $\gamma_{c}(t, x)$ are the known functions, while the functions $S_{b}(t, x)$ defining free boundary surfaces must be found. We assume that all functions arising in (4) -(7) are sufficiently smooth so that a classical solution exists for this BVP.

Consider an $N$-parameter (local) Lie group $G_{N}$ of point transformations of variables $(t, x, u)$ in the Euclidean space $\mathbb{R}^{n+2}$ (open subset of $\mathbb{R}^{n+2}$ ), which is given by equations

$$
t^{*}=T(t, x, \varepsilon), \quad x^{*}=X(t, x, \varepsilon), \quad u_{i}^{*}=U_{i}(t, x, u, \varepsilon), i=1, \ldots, n,
$$

where $\varepsilon=\left(\varepsilon_{1}, \varepsilon_{2}, \ldots, \varepsilon_{N}\right)$ are the group parameters. According to the general Lie group theory, one may construct the corresponding $N$-dimensional Lie algebra $L_{N}$ with the basic generators

$$
X_{\alpha}=\xi_{\alpha}^{0} \frac{\partial}{\partial t}+\xi_{\alpha}^{1} \frac{\partial}{\partial x}+\eta_{\alpha}^{1} \frac{\partial}{\partial u^{1}}+\ldots+\eta_{\alpha}^{n} \frac{\partial}{\partial u^{n}}, \alpha=1,2, \ldots, N,
$$

where $\xi_{\alpha}^{0}=\left.\frac{\partial T(t, x, \varepsilon)}{\partial \varepsilon_{\alpha}}\right|_{\varepsilon=0}, \xi_{\alpha}^{1}=\left.\frac{\partial X(t, x, \varepsilon)}{\partial \varepsilon_{\alpha}}\right|_{\varepsilon=0}, \eta_{\alpha}^{i}=\left.\frac{\partial U_{i}(t, x, u, \varepsilon)}{\partial \varepsilon_{\alpha}}\right|_{\varepsilon=0}$. 
Consider the Lie algebra $L_{N}$ in the extended space $\mathbb{R}^{n+q+2}$ of the variables $(t, x, u, S)$, where $S=\left(S_{1}, \ldots, S_{q}\right)$ are new dependent variables with respect to $t$ and $x$. In the extended space $\mathbb{R}^{n+q+2}$, the Lie group $\widetilde{G}_{N}$ corresponding to this algebra is given by transformations

$$
t^{*}=T(t, x, \varepsilon), x^{*}=X(t, x, \varepsilon), u_{i}^{*}=U_{i}(t, x, u, \varepsilon), S_{b}^{*}=S_{b}(t, x), i=1, \ldots, n, b=1, \ldots, q .
$$

Now we propose a new definition, which is based on the standard definition of differential equation invariance as an invariant manifold $\mathcal{M}$ in the relevant space of variables and on the prolongation theory [3].

Definition 2 A boundary value problem (4)-(7) is called to be invariant with respect to the Lie group $\widetilde{G}_{N}(\underline{10})$ if:

(a) the manifold determined by Eqs. (4) in the space of variables $\left(t, x, u, \ldots, u^{(k)}\right)$ is invariant with respect to the $k$ th-order prolongation of the group $G_{N}$;

(b) each manifold determined by conditions (5) with any fixed number a is invariant with respect to the $k_{a}$ th-order prolongation of the group $G_{N}$ in the space of variables $\left(t, x, u, \ldots, u^{\left(k_{a}\right)}\right)$, where $k_{a}=\max \left\{k_{a}^{j}, j=1, \ldots, n_{a}\right\}$;

(c) each manifold determined by conditions (6) with any fixed number $b$ is invariant with respect to the $k_{b}$ th-order prolongation of the group $\widetilde{G}_{N}$ in the space of variables $\left(t, x, u, \ldots, u^{\left(k_{b}\right)}, S_{b}, \frac{\partial S_{b}}{\partial t}, \frac{\partial S_{b}}{\partial x}\right)$, where $k_{b}=\max \left\{k_{b}^{l}, l=1, \ldots, n_{b}\right\} ;$

(d) each manifold determined by conditions (7) with any fixed number $c$ is invariant with respect to the $k_{c}$ th-order prolongation of the group $G_{N}$ in the space of variables $\left(t, x, u, \ldots, u^{\left(k_{c}\right)}\right)$, where $k_{c}=\max \left\{k_{c}^{m}, m=1, \ldots, n_{c}\right\}$.

Definition 3 The functions $u_{i}=\Phi_{i}(t, x), i=1, \ldots, n$ and $S_{b}=\Psi_{b}(t, x), b=1, \ldots, q$ form an invariant solution of BVP (4)-(7) corresponding to the Lie group (10) if:

(i) the functions $u_{i}=\Phi_{i}(t, x)$ and $S_{b}=\Psi_{b}(t, x)$ satisfy equations (4)-(7);

(ii) the manifold $\mathcal{M}=\left\{u_{i}=\Phi_{i}(t, x), i=1, \ldots, n ; S_{b}=\Psi_{b}(t, x), b=1, \ldots, q\right\}$ is an invariant manifold of the Lie group (10).

Remark 1 Definition 2 can be generalized on more general systems (including hyperbolic and elliptic those) and boundary conditions containing high-order derivatives for $S_{b}(t, x)$.

Remark 2 If free boundaries are given in the form $x=S_{b}(t)$, where $b=1, \ldots, q$ then we simply take $S_{b}(t, x) \equiv x-S_{b}(t)=0$. On the other hand, one can formulate a definition of Lie invariance for BVPs with such form of the free boundaries (see, e.g., [36]). However, the form used in Definition 2 is more convenient for generalization on multidimensional BVPs.

Now we present a non-trivial result to illustrate Definition 2. Let us consider the nonlinear BVP modeling the heat transfer in semi-infinite solid rod assuming that thermal diffusivity depends on temperature and the rod is exposed to a periodical flux of energy at the left endpoint. It should be noted that we neglect the initial distribution of the temperature in the 
Table 1: Lie invariance of BVP (11)-(13)

\begin{tabular}{ccccl}
\hline \hline no & $k$ & $\gamma$ & $q_{0}$ & Lie groups of invariance \\
\hline 1. & $\forall$ & $\forall$ & 0 & $t^{*}=t e^{2 \varepsilon_{2}}+\varepsilon_{1}, x^{*}=x e^{\varepsilon_{2}+k \varepsilon_{3}}, u^{*}=u e^{2 \varepsilon_{3}}$ \\
2. & $\forall$ & 0 & $\forall,\left(q_{0} \neq 0\right)$ & $t^{*}=t e^{(k+2) \varepsilon_{2}}+\varepsilon_{1}, x^{*}=x e^{(k+1) \varepsilon_{2}}, u^{*}=u e^{\varepsilon_{2}}$ \\
3. & -2 & $\forall,(\gamma \neq 0)$ & $\forall,\left(q_{0} \neq 0\right)$ & $t^{*}=t, x^{*}=x e^{-\varepsilon_{1}}, u^{*}=u e^{\varepsilon_{1}}$ \\
\hline \hline
\end{tabular}

rod, i.e., consider the process on the stage when the heat transfer already started. Thus the nonlinear BVP reads as

$$
\begin{aligned}
\frac{\partial u}{\partial t} & =\frac{\partial}{\partial x}\left(d(u) \frac{\partial u}{\partial x}\right), t>0,0<x<+\infty \\
x & =0: d(u) \frac{\partial u}{\partial x}=q_{0} \cos (\gamma t), t>0 \\
x & =+\infty: u=0, t>0
\end{aligned}
$$

where $u(t, x)$ is an unknown temperature field, $d(u)$ is a thermal diffusivity coefficient, $q_{0} \cos (\gamma t)$ is an energy flux. We assume that all functions arising in (11)-(13) are sufficiently smooth, so that a classical solution exists for this BVP.

Here we restrict ourselves to the case when the thermal diffusivity coefficient depends on the temperature as a power low, i.e. $d(u)=u^{k}$, where $k \in \mathbb{R}, k \neq 0$ (in the case $k=0$, the problem is liner and can be solved by classical methods, see, e.g., 41]). Notably, equation (11) with $d(u)=u^{k}$ presents the most interesting cases of Lie symmetry invariance [1]. In the case $d(u)=u^{k}\left(k \in \mathbb{R}, k \neq-\frac{4}{3}\right)$, it admits a four-dimensional Lie group. The corresponding algebra $L_{4}$ possesses the basic operators $\left\langle\partial_{t}, \partial_{x}, 2 t \partial_{t}+x \partial_{x}, k x \partial_{x}+2 u \partial_{u}\right\rangle$. These operators generate the one-parameter Lie groups

$$
\begin{aligned}
& T_{1}: t^{*}=t+\varepsilon_{1}, \quad x^{*}=x, \quad u^{*}=u \\
& T_{2}: t^{*}=t, \quad x^{*}=x+\varepsilon_{2}, \quad u^{*}=u \\
& T_{3}: t^{*}=t e^{2 \varepsilon_{3}}, \quad x^{*}=x e^{\varepsilon_{3}}, \quad u^{*}=u \\
& T_{4}: t^{*}=t, \quad x^{*}=x e^{k \varepsilon_{4}}, \quad u^{*}=u e^{2 \varepsilon_{4}}
\end{aligned}
$$

respectively (hereafter $\varepsilon_{i}, i=1, \ldots, 4$ are arbitrary group parameters). If $k=-\frac{4}{3}$, then the additional conformal generator $\left\langle x^{2} \partial_{x}-3 x u \partial_{u}\right\rangle$ occurs, which extends $L_{4}$ to the five-dimensional Lie algebra $L_{5}$. Thus, the case $k=-\frac{4}{3}$ should be examined separately.

Theorem 1 All possible Lie groups of invariance of the nonlinear BVP (11)-(13) with $d(u)=$ $u^{k}, k \neq 0$ for any constants $q_{0}$ and $\gamma$ are presented in Table 1.

Proof. On the first step of the proof we will consider BVP (11)-(13) with the constant energy flux $q(t)=q_{0}$, i.e. $\gamma=0$. Let us study the case of arbitrary power $k \neq-\frac{4}{3}$. First of all, we consider the one-parameter Lie groups (14)-(17) generated by the basic operators of $L_{4}$. One easily notes that BVP (11)-(13) is invariant with respect to the Lie group $T_{1}$ and isn't invariant under the Lie group $T_{2}$ since the boundary curve $x=0$ isn't invariant with respect to the transformations (15). 
According to item (b) of Definition 2, the boundary condition (12) is invariant with respect to the one-parameter group $T_{3}$, if the manifold $\mathcal{M}=\left\{x=0, u^{k} \frac{\partial u}{\partial x}-q_{0}=0\right\}$ satisfies the conditions

$$
\left.x^{*}\right|_{\mathcal{M}}=0,\left(u^{*}\right)^{k} \frac{\partial u^{*}}{\partial x^{*}}-\left.q_{0}\right|_{\mathcal{M}}=0
$$

The first equation of (18) is an identity, while the second equation leads to the expression $q_{0} e^{-\varepsilon_{3}}=q_{0}$, which immediately gives

$$
q_{0} \equiv 0 .
$$

The invariance of (13) under the one-parameter group $T_{3}$ is obvious. Thus, BVP (11)-(13) is invariant with respect to the Lie group $T_{3}$ if and only if the restriction (19) takes place.

Dealing in a similar way with the Lie group $T_{4}$, we obtain that BVP (11)-(13) is invariant with respect to $T_{4}$ only in two cases: $q_{0} \neq 0, k=-2$ and $q_{0}=0, k \in \mathbb{R}$. Indeed, according to item (b) of Definition 2, the boundary condition (12) is invariant with respect to $T_{4}$, if conditions (18) are satisfied on the manifold $\mathcal{M}$. Hence, we arrive at the restriction

$$
q_{0} e^{(k+2) \varepsilon_{4}}=q_{0}
$$

which immediately leads to $k=-2$ provided $q_{0} \neq 0$, and $k \in \mathbb{R}$ if $q_{0}=0$. The invariance of (13) under the one-parameter Lie group $T_{4}$ is evident.

Taking into account the restrictions considered above on $q_{0}$ and $k$, one concludes that BVP (11) -(13) is invariant with respect to the two-parameter Lie group $T_{1} \circ T_{4}$ iff $k=-2, q_{0} \neq 0$ and with respect to the three-parameter Lie group $T_{1} \circ T_{3} \circ T_{4}$ iff $k \in \mathbb{R}, q_{0}=0$ (it is exactly case 1 of Table 1 ).

To find other Lie groups of invariance, one needs to consider a linear combination of the basic operators of $L_{4}$ excepting the operator $\partial_{t}$ (we remind that the BVP is invariant under the Lie group $T_{1}$ for arbitrary $q_{0}$ and $k$ )

$$
X=2 \lambda_{3} t \partial_{t}+\left(\lambda_{2}+\left(\lambda_{3}+k \lambda_{4}\right) x\right) \partial_{x}+2 \lambda_{4} u \partial_{u},
$$

where $\lambda_{i}, i=2, \ldots, 4$ are arbitrary parameters and at least two of them are non-zero. If $\lambda_{3}=0$, then one arrives only at the results obtained above for the Lie group $T_{4}$, if $\lambda_{4}=0$ then the result obtained above for the Lie group $T_{3}$ is recovered. If $\lambda_{3} \lambda_{4} \neq 0$ then two possibilities occur: $\lambda_{3}+k \lambda_{4} \neq 0$ and $\lambda_{3}+k \lambda_{4}=0$. Consider the case $\lambda_{3}+k \lambda_{4} \neq 0$ when operator (21) generates the Lie group

$$
T_{a}: t^{*}=t e^{2 \lambda_{3} \varepsilon_{a}}, x^{*}=x e^{\left(\lambda_{3}+k \lambda_{4}\right) \varepsilon_{a}}+\frac{\lambda_{2}}{\lambda_{3}+k \lambda_{4}}\left(e^{\left(\lambda_{3}+k \lambda_{4}\right) \varepsilon_{a}}-1\right), u^{*}=u e^{2 \lambda_{4} \varepsilon_{a}} .
$$

Clearly, the boundary condition (13) is invariant with respect to $T_{a}$. Boundary conditions (12) is invariant under $T_{a}$, if and only if conditions (18) are satisfied. Now we realize that the first equation of (18) leads to the requirement $\lambda_{2}=0$ while the second equation of (18) gives

$$
q_{0} e^{\left((k+2) \lambda_{4}-\lambda_{3}\right) \varepsilon_{a}}=q_{0}
$$

Since $\lambda_{3} \lambda_{4} \neq 0$, one immediately obtains $\frac{\lambda_{3}}{\lambda_{4}}=k+2$ provided $q_{0} \neq 0$ and $k \neq-2$. If $q_{0}=0$ then we immediately arrive at case 1 from Table 1 . On the other hand, the Lie group $T_{1} \circ T_{a}$ transforms into the group $T_{1} \circ T_{4}$, when $k=-2$. Thus, we can conclude that the BVP under study is invariant with respect to the two-parameter Lie group $T_{1} \circ T_{a}$ if and only if

$$
q_{0} \neq 0, \quad \frac{\lambda_{3}}{\lambda_{4}}=k+2
$$


It is exactly case 2 of Table 1 . The examination of the case $\lambda_{3}+k \lambda_{4}=0$ leads to case 2 with $k=-1$. Thus, the invariance of BVP (11)-(13) with $k \neq-\frac{4}{3}$ is completely examined.

Now we examine the special case $k=-\frac{4}{3}$. One easily checks that the one-parameter groups (with $k=-\frac{4}{3}$ ) listed in cases $1-2$ of Table 1 are the groups of invariance of BVP (11) $-(13)$ (with $k=-\frac{4}{3}$ ) under the same restrictions on the constant $q_{0}$.

Thus, we need to examine whether the BVP in question can be invariant with respect to a Lie group corresponding to any liner combination of the basic operators of $L_{5}$

$$
X=2 \lambda_{3} t \partial_{t}+\left(\lambda_{2}+\left(\lambda_{3}+k \lambda_{4}\right) x+\lambda_{5} x^{2}\right) \partial_{x}+\left(2 \lambda_{4}-3 \lambda_{5} x\right) u \partial_{u}, \quad \lambda_{5} \neq 0 .
$$

To avoid cumbersome formulae, we consider the one-parameter Lie group corresponding to the pure conformal operator

$$
T_{5}: t^{*}=t, \quad x^{*}=\frac{x}{1-\varepsilon_{5} x}, \quad u^{*}=\left(1-\varepsilon_{5} x\right)^{3} u .
$$

Let us study the invariance of the boundary condition (13). According to item (d) of Definition 2 , the following equalities should take place

$$
\left.x^{*}\right|_{\mathcal{N}}=+\infty,\left.\quad u^{*}\right|_{\mathcal{N}}=0,
$$

where $\mathcal{N}=\{x=+\infty, u=0\}$. However, $\left.x^{*}\right|_{\mathcal{N}}=-\frac{1}{\varepsilon_{5}}$. Thus, the contradiction is obtained and we conclude that BVP (11)-(13) with $k=-\frac{4}{3}$ isn't invariant under $T_{5}$.

In a quite similar way, one may show that the boundary condition (13) isn't invariant under any Lie group corresponding to operator (25).

Finally, to complete the proof, we must consider the case, when the flux of energy has periodical form, i.e. $\gamma \neq 0$. Obviously, $q_{0}$ must be nonzero, otherwise we obtain the case examined above. Since calculations are quite similar to the case $\gamma=0$ (an analog of formula (20) plays a crucial role to derive the special power $k=-2$ ), we present the result: BVP (11) -(13) with the periodic energy flux $q(t)=q_{0} \cos (\gamma t)$ is invariant only with respect to the one-parameter Lie group $T_{4}$ with $k=-2$ (case 3 from Table 1 ).

The proof is now completed.

Remark 3 Theorem 1 highlights that Definition 2 is non-trivial because the power $k=-2$ isn't a special one for Lie invariance of standard nonlinear heat equation (11) with $d(u)=u^{k}$, however, $k=-2$ is the special power if one looks for Lie invariance of BVP (11)-(13).

\section{Lie invariance of a class of (1+1)-dimensional nonlin- ear BVPs of Stefan type}

In this section we consider a class of $(1+1)$-dimensional BVPs of Stefan type used to describe melting and evaporation of materials in the case that their surface is exposed to a powerful flux of energy. Such problems also arise in mathematical modeling of other processes in biology (tumor growth) and physics (crystal growth). The class of BVPs after some simplifications (like using the Goodman substitution to transform the basic equations to the standard heat equations) can be written as follows

$$
\frac{\partial u}{\partial t}=\frac{\partial}{\partial x}\left(d_{1}(u) \frac{\partial u}{\partial x}\right),
$$




$$
\begin{aligned}
\frac{\partial v}{\partial t}= & \frac{\partial}{\partial x}\left(d_{2}(v) \frac{\partial v}{\partial x}\right) \\
& S_{1}(t, x)=0: d_{1}(u) \frac{\partial u}{\partial x}=H_{1}(u) V_{1}-q(t, u), V_{1}=h(t, u) \\
& S_{2}(t, x)=0: d_{2}\left(v_{m}\right) \frac{\partial v}{\partial x}=d_{1}\left(u_{m}\right) \frac{\partial u}{\partial x}+H_{2}\left(v_{m}\right) V_{2}, u=u_{m}, v=v_{m} \\
& x=+\infty: v=v_{\infty}
\end{aligned}
$$

where $u(t, x)$ and $v(t, x)$ are the unknown temperature fields; $S_{k}(t, x), k=1,2$ are the unknown functions, which determine the phase division boundaries (they can be also presented in the form $\left.S_{k}(t, x)=x-s_{k}(t)\right) ; V_{k}(t, x)=-\frac{\partial S_{k}}{\partial t} / \frac{\partial S_{k}}{\partial x} k=1,2$ are the phase division boundary velocities; $q(t, u)$ is the known strictly positive function presenting the energy flux being absorbed by the material; $h(t, u)$ is the known non-negative function describing dynamics of evaporation process; $H_{k}, k=1,2$ are the known strictly positive function presenting specific heat values per unit volume of liquid and solid phases. The parameters $u_{m}, v_{m}$ and $v_{\infty}$ are assumed to known, moreover, $v_{m} \neq v_{\infty}$.

Here Eqs. (28) and (29) describe the heat transfer process in liquid and solid phases, the boundary conditions (30) present evaporation dynamics on the surface $S_{1}(t, x)=0$, and the boundary conditions (31) are the famous Stefan conditions on the surface $S_{2}(t, x)=0$ dividing the liquid and solid phases. Assuming that the liquid phase thickness is considerably less than the solid phase thickness, one may use the Dirichlet condition (32). It should be stressed that we neglect the initial distribution of the temperature in the solid phase and consider the process on the stage when two phases take already place.

One may claim that formulae (28) -(32) present a class of BVPs with moving boundaries and take into account a number of different situations, which occur in the melting and evaporation processes. Setting $q(t, u)=$ const, $V_{1}=$ const $\Phi(t)$ and $h(t, u)=\Phi(t) u$, where $\Phi(t)$ is a correctlyspecified function, one obtains the problem, which is the most typical, see, e.g., [30]. In the case of a process when surfaces are exposed to very powerful periodic laser pulses these functions take complicated forms [39].

The BVP obtained is based on the standard nonlinear heat equations. Lie symmetry of noncoupled system (28)-(29) can be easily derived using the determining equations from paper [42], where reaction-diffusion systems of more general form have been investigated. Now we formulate a theorem, which gives complete information on Lie symmetry of this system.

Theorem 2 All possible maximal algebras of invariance (up to equivalent representations generated by transformations of the form (33)) of the system (28) and (29) for any fixed vectors $\left(d_{1}, d_{2}\right)$ with strictly positive functions $d_{1}(u)$ and $d_{2}(v)$ are presented in Table 2. Any other system of the form (28) and (29) is reduced to one of those with diffusivities from Table 2 by an equivalence transformation of the form

$$
t \rightarrow e_{0} t+t_{0}, \quad x \rightarrow e_{1} x+x_{0}, \quad u \rightarrow e_{2} u+u_{0}, \quad v \rightarrow e_{3} v+v_{0}
$$

where $e_{i} \neq 0(i=0, \ldots, 3), t_{0}, x_{0}, u_{0}$, and $v_{0}$ are arbitrary parameters.

Remark 4 In the case of linear system (28)-(29) with $k_{1}=k_{2}$ (see case 9 of Table 2), the Lie algebra extension occurs by the operators $v \partial_{u}$ and $u \partial_{v}$. However, BVP (28)-(32) with $d_{1}(u)=d_{2}(v)=k_{1}$ is rather artificial from physical point because diffusivities of solid and liquid phases must be different. Thus, we don't consider this case below. 
Table 2: Lie algebras of NHEs system (28)-(29). (Here $k_{1}, k_{2}, m$ and $n$ are arbitrary non-zero constants; while $\alpha(t, x)$ and $\beta(t, x)$ are arbitrary solutions of the linear heat equations $\alpha_{t}=$ $k_{1} \alpha_{x x}$ and $\beta_{t}=k_{2} \beta_{x x}$, respectively.)

\begin{tabular}{cccl}
\hline \hline no & $d_{1}(u)$ & $d_{2}(v)$ & Basic operators of MAI \\
\hline 1. & $\forall$ & $\forall$ & $A=\left\langle\partial_{t}, \partial_{x}, 2 t \partial_{t}+x \partial_{x}\right\rangle$ \\
2. & $k_{1}$ & $\forall$ & $A, u \partial_{u}, \alpha(t, x) \partial_{u}$ \\
3. & $\forall$ & $k_{2}$ & $A, v \partial_{v}, \beta(t, x) \partial_{v}$ \\
4. & $e^{u}$ & $e^{v}$ & $A, x \partial_{x}+2 \partial_{u}+2 \partial_{v}$ \\
5. & $e^{u}$ & $v^{m}$ & $A, x \partial_{x}+2 \partial_{u}+\frac{2}{m} v \partial_{v}$ \\
6. & $u^{n}$ & $e^{v}$ & $A, x \partial_{x}+\frac{2}{n} u \partial_{u}+2 \partial_{v}$ \\
7. & $u^{n}$ & $v^{m}$ & $A, x \partial_{x}+\frac{2}{n} u \partial_{u}+\frac{2}{m} v \partial_{v}$ \\
8. & $u^{-\frac{4}{3}}$ & $v^{-\frac{4}{3}}$ & $A, x \partial_{x}-\frac{3}{2} u \partial_{u}-\frac{3}{2} v \partial_{v}, x^{2} \partial_{x}-3 x u \partial_{u}-3 x v \partial_{v}$ \\
9. & $k_{1}$ & $k_{2}$ & $A, u \partial_{u}, v \partial_{v}, \alpha(t, x) \partial_{u}, \beta(t, x) \partial_{v}, 2 t \partial_{x}-x\left(\frac{1}{k_{1}} u \partial_{u}+\frac{1}{k_{2}} v \partial_{v}\right)$, \\
& & & $4 t x \partial_{x}+4 t^{2} \partial_{t}-\frac{1}{k_{1}}\left(x^{2}+2 k_{1} t\right) u \partial_{u}-\frac{1}{k_{2}}\left(x^{2}+2 k_{2} t\right) v \partial_{v}$ \\
\hline \hline
\end{tabular}

Table 3: Lie invariance of BVP (28)-(32)

\begin{tabular}{cccl}
\hline \hline no & $q(t, u)$ & $h(t, u)$ & Lie groups of invariance \\
\hline 1. & $\forall$ & $\forall$ & $t^{*}=t, x^{*}=x+\varepsilon, u^{*}=u, v^{*}=v, S_{1}^{*}=S_{1}, S_{2}^{*}=S_{2}$ \\
2. & $q(u)$ & $h(u)$ & $t^{*}=t+\varepsilon_{1}, x^{*}=x+\varepsilon_{2}, u^{*}=u, v^{*}=v, S_{1}^{*}=S_{1}, S_{2}^{*}=S_{2}$ \\
3. & $\frac{q(u)}{\sqrt{t}}$ & $\frac{h(u)}{\sqrt{t}}$ & $t^{*}=t e^{2 \varepsilon_{1}}, x^{*}=x e^{\varepsilon_{1}}+\varepsilon_{2}, u^{*}=u, v^{*}=v, S_{1}^{*}=S_{1}, S_{2}^{*}=S_{2}$ \\
\hline \hline
\end{tabular}

Remark 5 If one takes into account the trivial discrete transformations $t \rightarrow t, x \rightarrow x, u \rightarrow v$, and $v \rightarrow u$, then cases 2 and 3, 5 and 6 arising in Table 2 are equivalent. However, the class of BVPs (28)-(32) isn't invariant under these transformations because of boundary conditions (30) and (32). Thus, we don't take into account them in what follows.

Using the set of equivalence transformations (33), we can straightforwardly extend one to the relevant set for BVP (28)-(32) by adding the identical transformations for the variables $S_{k}(t, x)$. Direct calculations show that the most general form of those is

$$
t \rightarrow e_{0} t+t_{0}, \quad x \rightarrow e_{1} x+x_{0}, \quad u \rightarrow e_{2} u+u_{0}, \quad v \rightarrow e_{3} v+v_{0}, \quad S_{1} \rightarrow S_{1}, \quad S_{2} \rightarrow S_{2} .
$$

where $e_{i} \neq 0(i=0, \ldots, 3), t_{0}, x_{0}, u_{0}$, and $v_{0}$ are arbitrary parameters $\left(e_{1}>0\right)$.

Now we formulate the main result of this section.

Theorem 3 BVP (28)-(32) with any smooth functions $d_{1}(u), d_{2}(v), q(t, u), h(t, u)$ and $H_{1}(u)$ is invariant under the one-parameter Lie group presented in case 1 of Table 3. All possible 
extensions of this Lie group invariance (up to equivalent representations generated by equivalence transformations of the form (34)) depend only on the form of the functions $q(t, u)$ and $h(t, u)$, and are presented in cases 2 and 3 of Table 3. Any other BVP of the form (28)-(32) is invariant under two-parameter Lie group is reduced by transformations (34) to one of those with the functions $q(t, u)$ and $h(t, u)$ from Table 3.

Proof. According to Definition 2 and Theorem 2 we need to examine the nine different cases listed in Table 2. It turns out that the examination of the first case, when the functions $d_{1}(u)$ and $d_{2}(v)$ are arbitrary, leads to the main result of the theorem presented in Table 3.

Let us consider the one-parameter Lie groups corresponding to the basic operators of algebra A. Obviously, BVP (28)-(32) with arbitrary given functions is invariant under the group of space translations generated by the operator $P_{x}=\partial_{x}$ and this is listed in the first case of Table 3. Since any linear combination $\lambda_{1} P_{t}+\lambda_{2} D$ of other two operators is equivalent (up to transformations (34)) either to $P_{t}=\partial_{t}$ (if $\lambda_{2}=0$ ) or to $D=2 t \partial_{t}+x \partial_{x}$ (if $\lambda_{2} \neq 0$ ), we should separately examine these two operators.

Now we apply Definition 2 to $D$. Taking into account that BVP (28)-(32) has two free boundaries, we construct the extended Lie group $\widetilde{T}_{D}$ corresponding to the operator $D$ :

$$
\widetilde{T}_{D}: t^{*}=t e^{2 \varepsilon_{1}}, x^{*}=x e^{\varepsilon_{1}}, u^{*}=u, v^{*}=v, S_{1}^{*}=S_{1}, S_{2}^{*}=S_{2} .
$$

According to item (c), the boundary conditions (30) are invariant with respect to the group $\widetilde{T}_{D}$, if the manifold $\mathcal{M}=\left\{S_{1}(t, x)=0, d_{1}(u) \frac{\partial u}{\partial x}=H_{1}(u) V_{1}-q(t, u), V_{1}-h(t, u)=0\right\}$ satisfies the conditions

$$
\left.S_{1}^{*}\right|_{\mathcal{M}}=0, d_{1}\left(u^{*}\right) \frac{\partial u^{*}}{\partial x^{*}}-H_{1}\left(u^{*}\right) V_{1}^{*}+\left.q\left(t^{*}, u^{*}\right)\right|_{\mathcal{M}}=0, \quad V_{1}^{*}-\left.h\left(t^{*}, u^{*}\right)\right|_{\mathcal{M}}=0 .
$$

Taking into account (35), one finds

$$
\frac{\partial u^{*}}{\partial x^{*}}=e^{-\varepsilon_{1}} \frac{\partial u}{\partial x}, \frac{\partial v^{*}}{\partial x^{*}}=e^{-\varepsilon_{1}} \frac{\partial v}{\partial x}, V_{k}^{*}=e^{-\varepsilon_{1}} V_{k}, k=1,2,
$$

so that the second and third equations of (36) produce the equations

$$
e^{\varepsilon_{1}} q\left(t e^{2 \varepsilon_{1}}, u\right)=q(t, u) \quad e^{\varepsilon_{1}} h\left(t e^{2 \varepsilon_{1}}, u\right)=h(t, u),
$$

to find the functions $q(t, u)$ and $h(t, u)$. Solving (38) one obtains

$$
q(t, u)=\frac{q(u)}{\sqrt{t}}, \quad h(t, u)=\frac{h(u)}{\sqrt{t}},
$$

where $q(u)$ and $h(u)$ are arbitrary smooth functions. The invariance criterium of the boundary conditions (31) for $\widetilde{T}_{D}$ is fulfilled for arbitrary parameters arising in (31), while the invariance of condition (32) under $T_{D}$ is obvious. Thus, BVP (28)-(32) is invariant with respect to the Lie group $\widetilde{T}_{D}$ if and only if restrictions (39) take place. This is exactly listed in case 3 of Table 3 .

In a quite similar way one can show, that the BVP under study is invariant with respect to the extended Lie group corresponding to the operator $P_{t}=\partial_{t}$ if and only if the restrictions on

$$
q(t, u)=q(u) \text { and } h(t, u)=h(u),
$$

take place, and this is what exactly listed in case 2 of Table 3. 
Much more cumbersome calculations are needed to show that there are no any new Lie group invariance for BVP (28) -(32) nevertheless there are eight special cases listed in Table 2, which lead to the extensions of MAI of the basic equations (28).

Let us consider case 2 of Table 2. Firstly, we check the invariance of BVP (28)-(32) with respect to the one-parameter extended Lie groups corresponding to the operators $X_{1}=u \partial_{u}$ and $X_{2}=\alpha(t, x) \partial_{u}$ :

$$
\widetilde{T}_{1}: t^{*}=t, x^{*}=x, u^{*}=u e^{\varepsilon_{1}}, v^{*}=v, S_{1}^{*}=S_{1}, S_{2}^{*}=S_{2},
$$

and

$$
\widetilde{T}_{2}: t^{*}=t, x^{*}=x, u^{*}=u+\alpha(t, x) \varepsilon_{2}, v^{*}=v, S_{1}^{*}=S_{1}, S_{2}^{*}=S_{2} .
$$

According to item (c) of Definition 2, the boundary conditions (31) are invariant with respect to the group $\widetilde{T}_{1}$, if the conditions

$$
\left.S_{2}^{*}\right|_{\mathcal{N}}=0, d_{2}\left(v_{m}\right) \frac{\partial v^{*}}{\partial x^{*}}-d_{1}\left(u_{m}\right) \frac{\partial u^{*}}{\partial x^{*}}-\left.H_{2}\left(v_{m}\right) V_{2}^{*}\right|_{\mathcal{N}}=0, u^{*}-\left.u_{m}\right|_{\mathcal{N}}=0, v^{*}-\left.v_{m}\right|_{\mathcal{N}}=0
$$

are satisfied, where the manifold

$$
\mathcal{N}=\left\{S_{2}(t, x)=0, d_{2}\left(v_{m}\right) \frac{\partial v}{\partial x}-d_{1}\left(u_{m}\right) \frac{\partial u}{\partial x}-H_{2}\left(v_{m}\right) V_{2}=0, u-u_{m}=0, v-v_{m}=0\right\} .
$$

Taking into account (41) and the second equation of (43), we arrive at the requirement

$$
\frac{\partial u}{\partial x}=\frac{\partial u}{\partial x} e^{\varepsilon_{1}} \Rightarrow \varepsilon_{1}=0 .
$$

Similarly, one easily checks that the boundary conditions (31) isn't invariant with respect to the Lie group $\widetilde{T}_{2}$, too. Indeed, to satisfy the third equation of (43), one obtains the requirement

$$
\alpha(t, x) \varepsilon_{2}=0 \Rightarrow \varepsilon_{2}=0 .
$$

Let us now examine the invariance of BVP (28)-(32) with respect to an extended Lie group $\widetilde{T}_{c}$ corresponding to a liner combination of operators $P_{t}, D, X_{1}$, and $X_{2}$, i.e.

$$
X_{c}=\left(\lambda_{1}+2 \lambda_{2} t\right) \partial_{t}+\lambda_{2} x \partial_{x}+\left(\lambda_{3} u+\lambda_{4} \alpha(t, x)\right) \partial_{u},
$$

where $\lambda_{i}, i=1, \ldots, 4$ are arbitrary parameters and $\lambda_{3}^{2}+\lambda_{4}^{2} \neq 0$ (otherwise the operator $\lambda_{1} P_{t}+\lambda_{2} D$ is obtained). Having transformations (34), we can put $\lambda_{1}=0$ and $\lambda_{2}=1$ in (46) so that the operator takes the form

$$
X_{c}=2 t \partial_{t}+x \partial_{x}+\left(\lambda_{3} u+\lambda_{4} \alpha(t, x)\right) \partial_{u}, \lambda_{3}^{2}+\lambda_{4}^{2} \neq 0 .
$$

The corresponding Lie group $\widetilde{T}_{c}$ is

$$
\begin{aligned}
\widetilde{T}_{c}: t^{*} & =t e^{2 \varepsilon_{c}}, x^{*}=x e^{\varepsilon_{c}}, u^{*}=u e^{\lambda_{3} \varepsilon_{c}}+\lambda_{4} \int_{0}^{\varepsilon_{c}} \alpha\left(t e^{2 \tau}, x e^{\tau}\right) e^{\lambda_{3}\left(\tau-\varepsilon_{c}\right)} d \tau, v^{*}=v, \\
S_{1}^{*} & =S_{1}, S_{2}^{*}=S_{2} .
\end{aligned}
$$

Now we again show that boundary conditions (31) are not invariant under $\widetilde{T}_{c}$. In fact, the third equation of (43) gives the restriction

$$
\lambda_{4} \int_{0}^{\varepsilon_{c}} \alpha\left(t e^{2 \tau}, x e^{\tau}\right) e^{\lambda_{3}\left(\tau-\varepsilon_{c}\right)} d \tau=u_{m}\left(1-e^{\lambda_{3} \varepsilon_{c}}\right)
$$


so that the Lie group $\widetilde{T}_{c}$ can be written in the form

$$
\widetilde{T}_{c}: t^{*}=t e^{2 \varepsilon_{c}}, x^{*}=x e^{\varepsilon_{c}}, u^{*}=u e^{\lambda_{3} \varepsilon_{c}}+u_{m}\left(1-e^{\lambda_{3} \varepsilon_{c}}\right), v^{*}=v, S_{1}^{*}=S_{1}, S_{2}^{*}=S_{2} .
$$

Taking into account (50) and the second equation of (43), we arrive at the requirement

$$
\frac{\partial u}{\partial x}=\frac{\partial u}{\partial x} e^{\lambda_{3} \varepsilon_{c}} \Rightarrow \lambda_{3}=0,
$$

what leads to $\lambda_{4}=0$ (see (49) ). However, this contradicts to the assumption $\lambda_{3}^{2}+\lambda_{4}^{2} \neq 0$.

Hence, case 2 of Table 2 is completely examined. Cases 3 and 9 of Table 2 can be studied in a quite similar manner because each of them needs to examine groups (41) and (42).

Consider case 4 from Table 2 . Here the operator $X_{4}=x \partial_{x}+2 \partial_{u}+2 \partial_{v}$ arises, which generates the extended Lie group $\widetilde{T}_{4}$ :

$$
t^{*}=t, x^{*}=x e^{\varepsilon_{4}}, u^{*}=u+2 \varepsilon_{4}, v^{*}=v+2 \varepsilon_{4}, S_{1}^{*}=S_{1}, S_{2}^{*}=S_{2} .
$$

Applying Definition 2 to the boundary conditions (31) in the case of $\widetilde{T}_{4}$, equations (43) are again obtained. The third and fourth equations of (43) lead to the requirement

$$
u_{m}=u_{m}+2 \varepsilon_{4}, v_{m}=v_{m}+2 \varepsilon_{4},
$$

hence, $\varepsilon_{4}=0$. Thus, BVP (28)-(32) cannot be invariant with respect to the Lie group $\widetilde{T}_{4}$. Moreover, the same result is obtained if one examines any linear combination of operators $P_{t}$, $D$, and $X_{4}$, i.e.

$$
X=\left(\lambda_{1}+2 \lambda_{2} t\right) \partial_{t}+\left(\lambda_{2}+\lambda_{3}\right) x \partial_{x}+2 \lambda_{3} u \partial_{u}+2 \lambda_{3} v \partial_{v}, \quad \lambda_{3} \neq 0
$$

with $\lambda_{3} \neq 0$. Thus, we conclude, that the exponential diffusivities arising in case 4 of Table 2 don't lead to any new Lie groups of invariance of BVP (28)-(32). Cases 5 and 6 of Table 2 can be studied in a quite similar way.

Let us consider case 7 of Table 2, which needs separate examination. The extended Lie group $\widetilde{T}_{7}$ corresponding to the operator $X_{7}=x \partial_{x}+\frac{2}{n} u \partial_{u}+\frac{2}{m} v \partial_{v}$ is

$$
T_{7}: t^{*}=t, x^{*}=x e^{\varepsilon_{7}}, u^{*}=u e^{\frac{2}{n} \varepsilon_{7}}, v^{*}=v e^{\frac{2}{m} \varepsilon_{7}}, S_{1}^{*}=S_{1}, S_{2}^{*}=S_{2} .
$$

In order to the boundary conditions (31) be invariant under $\widetilde{T}_{7}$ equations (43) are again obtained. It turns out, equations (43) are fulfilled if $m>0, n>0, u_{m}=v_{m}=0$ and $H_{2}(0)=0$. Hence, we must apply item (d) of Definition 2 to the boundary conditions (32):

$$
\left.x^{*}\right|_{\mathcal{P}}=+\infty, \quad v^{*}-\left.v_{\infty}\right|_{\mathcal{P}}=0,
$$

where $\mathcal{P}=\left\{x=+\infty, v-v_{\infty}=0\right\}$. The group $\widetilde{T}_{7}$ transforms the second equation of (156) as follows

$$
v_{\infty} e^{\frac{2}{m} \varepsilon_{7}}-v_{\infty}=0
$$

hence, we arrive at the restriction $v_{\infty}=0$ (otherwise $\varepsilon_{7}=0$ ). Thus, the contradiction is obtained because, in the very beginning, we assumed $v_{m} \neq v_{\infty}$. One may check that the same result is obtained for any liner combination of the operators $P_{t}, D$, and $X_{7}$. Thus, case 7 of Table 2 is completely examined.

Case 8 of Table 2 can be treated in a quite similar way as we did in Theorem 1 (see formulae (26) and (27)).

The proof is now completed.

Finally, we note that theorem 2 from the recent paper [37] follows as a particular case from Theorem 3 (but not wise versa!), nevertheless Definition 2 was not used in [37]. 


\section{Symmetry reduction and invariant solutions of the class BVPs (28)-(32)}

In this section we consider symmetry reduction of BVPs of the form (28)-(32) to BVPs for systems of two ordinary differential equations and construct exact solutions for the reduced BVPs.

Operator $P_{x}$, corresponding to the invariance transformations in case 1 of Table 3 , leads to an ansatz, which doesn't depend on the space variable $x$. This contradicts to the free boundary surfaces and leads to non-physical solutions.

According to case 2 of Theorem 3, each BVPs belonging to the class under study for $q(t, u)=$ $q(u)$ and $h(t, u)=h(u)$ admits the two-dimensional Lie algebra with basic operators $P_{t}=\partial_{t}$ and $P_{x}=\partial_{x}$. Hence, it also admits the operator $X_{1}=\partial_{t}+\mu \partial_{x}, \mu \in \mathbb{R}$, which leads to the plane-wave ansatz

$$
u=u(\xi), v=v(\xi), S_{k}=S_{k}(\xi), \xi=x-\mu t,
$$

where $k=1,2$ and $\mu$ is an unknown velocity. Note, that ansatz (158) with $\mu=0$ leads to stationary solutions of the BVP in question. However, these solutions don't have essential physical sense so that will not studied hereafter.

In case 3 of Table 3, each BVP of the form (28)-(32) admits two-dimensional Lie algebra with basic operators $P_{x}$ and $D=2 t \partial_{t}+x \partial_{x}$. Obviously, that any linear combination of these operators is equivalent, up to transformations (34), to operator $D$, which generates the ansatz

$$
u=u(\omega), v=v(\omega), S_{k}=S_{k}(\omega), \omega=\frac{x}{\sqrt{t}}, \quad k=1,2 .
$$

Using ansätze (58) -(59) and taking into account the restrictions on the functions $q(t, u)$ and $h(t, u)$ (see Theorem 3 ) one can easy obtain the following theorems.

Theorem 4 Ansatz (58) reduces any nonlinear BVP of the form (28)-(32) with the coefficient restrictions $q(t, u)=q(u)$ and $h(t, u)=h(u)$ to the BVP for the second-order ODEs

$$
\begin{aligned}
& \frac{d}{d \xi}\left(d_{1}(u) \frac{d u}{d \xi}\right)+\mu \frac{d u}{d \xi}=0, \quad 0<\xi<\delta \\
& \frac{d}{d \xi}\left(d_{2}(v) \frac{d v}{d \xi}\right)+\mu \frac{d v}{d \xi}=0, \quad \xi>\delta \\
& \xi=0: d_{1}(u) \frac{d u}{d \xi}=H_{1}(u) \mu-q(u), \mu=h(u), \\
& \xi=\delta: d_{2}\left(v_{m}\right) \frac{d v}{d \xi}=d_{1}\left(u_{m}\right) \frac{d u}{d \xi}+H_{2}\left(v_{m}\right) \mu, u=u_{m}, v=v_{m}, \\
& \xi=+\infty: \quad v=v_{0},
\end{aligned}
$$

where $\delta$ and $\mu$ are to be determined parameters.

Theorem 5 Ansatz (59) reduces any nonlinear BVP of the form (28)-(32) with the coefficient restrictions $q(t, u)=\frac{q(u)}{\sqrt{t}}$ and $h(t, u)=\frac{h(u)}{\sqrt{t}}$ to the BVP for the second-order ODEs

$$
\begin{aligned}
& \frac{d}{d \omega}\left(d_{1}(u) \frac{d u}{d \omega}\right)+\frac{\omega}{2} \frac{d u}{d \omega}=0, \quad \omega_{1}<\omega<\omega_{2}, \\
& \frac{d}{d \omega}\left(d_{2}(v) \frac{d v}{d \omega}\right)+\frac{\omega}{2} \frac{d v}{d \omega}=0, \quad \omega>\omega_{2},
\end{aligned}
$$




$$
\begin{aligned}
& \omega=\omega_{1}: d_{1}(u) \frac{d u}{d \omega}=H_{1}(u) \frac{\omega_{1}}{2}-q(u), \frac{\omega_{1}}{2}=h(u), \\
& \omega=\omega_{2}: d_{2}\left(v_{m}\right) \frac{d v}{d \omega}=d_{1}\left(u_{m}\right) \frac{d u}{d \omega}+H_{2}\left(v_{m}\right) \frac{\omega_{2}}{2}, u=u_{m}, v=v_{m}, \\
& \omega=+\infty: v=v_{0},
\end{aligned}
$$

where $\omega_{1}$ and $\omega_{2}$ are to be determined parameters.

Let us consider BVP (60)-(64). It is well-known, that the system of nonlinear ordinary differential equations (60) and (61) with $\mu=0$ can be linearized by the Kirchhoff substitution. It turns out, this substitution can be generalized in the case $\mu \neq 0$ by introducing new independent variables 30 ]

$$
\begin{aligned}
& U=u-u_{m}, V=v-v_{0}, \\
& \xi=\int_{0}^{\eta} d_{1}\left(U+u_{m}\right) d \eta, \quad 0 \leq \xi \leq \delta, \\
& \xi=\delta+\int_{\delta^{*}}^{\eta} d_{2}\left(V+v_{0}\right) d \eta, \quad \xi \geq \delta,
\end{aligned}
$$

where the lower bonds of integration are chosen as $\left.\xi\right|_{\eta=0}=0$ and $\left.\xi\right|_{\eta=\delta^{*}}=\delta$.

Substituting (70) -(72) into BVP (60)-(64), one obtains the BVP for system of linear differential equations

$$
\begin{aligned}
& \frac{d^{2} U}{d \eta^{2}}+\mu \frac{d U}{d \eta}=0, \quad 0<\eta<\delta^{*} \\
& \frac{d^{2} V}{d \eta^{2}}+\mu \frac{d V}{d \eta}=0, \quad \eta>\delta^{*} \\
& \eta=0: \frac{d U}{d \eta}=H_{1}\left(U+u_{m}\right) \mu-q\left(U+u_{m}\right), U=h^{-1}(\mu)-u_{m} \\
& \eta=\delta^{*}: \frac{d V}{d \eta}=\frac{d U}{d \eta}+H_{2}\left(v_{m}\right) \mu, U=0, V=V_{m} \\
& \eta=+\infty: V=0
\end{aligned}
$$

where $h^{-1}$ is an inverse function to $h(u)$ (hereafter existence of this function is assumed), $V_{m}=v_{m}-v_{0}, \delta^{*}$ is new to be determined parameter.

To solve BVP (173) - (77) one needs to find the unknown functions $U=U(\eta)$ and $V=V(\eta)$ and the parameters $\mu$ and $\delta^{*}$. Since equations (73) and (74) are linear ordinary differential equations with constant coefficient their general solution is

$$
\begin{aligned}
& U=C_{1}+C_{2} e^{-\mu \eta}, 0 \leq \eta \leq \delta^{*}, \\
& V=C_{3}+C_{4} e^{-\mu \eta}, \eta \geq \delta^{*},
\end{aligned}
$$

where $C_{i}(i=1, \ldots, 4)$ are to be determined constants.

Substituting solution (78) into the second equation of the boundary conditions (75) and the second equation of (76), one finds the constants $C_{1}$ and $C_{2}$ :

$$
C_{1}=\left(h^{-1}(\mu)-u_{m}\right) \frac{e^{-\mu \delta^{*}}}{e^{-\mu \delta^{*}}-1}, C_{2}=\left(h^{-1}(\mu)-u_{m}\right) \frac{1}{e^{-\mu \delta^{*}}-1} .
$$


Similarly, the constants $C_{3}$ and $C_{4}$ can be found using the third equations from (76) and the boundary condition (77)

$$
C_{3}=0, C_{4}=V_{m} e^{\mu \delta^{*}} .
$$

Thus, using formulae (178)-(81), we construct the explicit formulae for the function $U=U(\eta)$ and $V=V(\eta)$ :

$$
\begin{aligned}
& U=\left(h^{-1}(\mu)-u_{m}\right) \frac{e^{\mu\left(\delta^{*}-\eta\right)}-1}{e^{\mu \delta^{*}}-1}, \\
& V=V_{m} e^{\mu\left(\delta^{*}-\eta\right)} .
\end{aligned}
$$

Finally, we need to specify the parameters $\mu$ and $\delta^{*}$. This can be done by substituting (82) and (83) into the first equations of the boundary conditions (75) and (76), and using the formulae

$$
\frac{d U}{d \eta}=-\mu\left(h^{-1}(\mu)-u_{m}\right) \frac{e^{\mu\left(\delta^{*}-\eta\right)}-1}{e^{\mu \delta^{*}}-1}, \frac{d V}{d \eta}=-\mu V_{m} e^{\mu\left(\delta^{*}-\eta\right)} .
$$

After the corresponding calculations, we arrive at the equations

$$
\begin{aligned}
& \left(h^{-1}(\mu)-u_{m}\right) \frac{e^{\mu \delta^{*}}}{e^{\mu \delta^{*}}-1}=\frac{q\left(h^{-1}(\mu)\right)}{\mu}-H_{1}\left(h^{-1}(\mu)\right), \\
& V_{m}=\left(h^{-1}(\mu)-u_{m}\right) \frac{1}{e^{\mu \delta^{*}}-1}-H_{2}\left(v_{m}\right) .
\end{aligned}
$$

Thus, equation (86) leads to the explicit formula for the parameter $\delta^{*}$, which corresponds to the thickness of liquid phase:

$$
\delta^{*}=\frac{1}{\mu} \log \left(1+\frac{h^{-1}(\mu)-u_{m}}{V_{m}+H_{2}\left(v_{m}\right)}\right) .
$$

Having (87), equations (85) produces immediately the transcendent equation to find the velocity $\mu$ :

$$
\frac{q\left(h^{-1}(\mu)\right)}{\mu}-H_{1}\left(h^{-1}(\mu)\right)-h^{-1}(\mu)=V_{m}-u_{m}+H_{2}\left(v_{m}\right) .
$$

Thus, formulae (82)-(83) give exact solution of problem (73)-(77), where the important parameters $\delta^{*}$ and $\mu$ are defined by expressions (87) and (88), respectively. It means that the exact solution of BVP (60) -(64) is obtained in implicit form. It should be stressed that this result is essential generalization of [30, where a particular case of BVP (28)-(32) was investigated.

Example. We use a model, which was formulated and investigated by numerical methods in [39]. The model describes the processes of heating, melting and evaporation of metals under the action at their surface of powerful laser pulses. Under the relevant assumptions it can be written as follows [39]

$$
\begin{aligned}
& \frac{\partial}{\partial x}\left(\lambda_{1}\left(T_{1}\right) \frac{\partial T_{1}}{\partial x}\right)=\rho c_{1}\left(T_{1}\right) \frac{\partial T_{1}}{\partial t}, \quad 0<s_{1}(t)<x<s_{2}(t), t>0, \\
& \frac{\partial}{\partial x}\left(\lambda_{2}\left(T_{2}\right) \frac{\partial T_{2}}{\partial x}\right)=\rho c_{2}\left(T_{2}\right) \frac{\partial T_{2}}{\partial t}, \quad x>s_{2}(t), t>0, \\
& \quad x=s_{1}(t): \lambda_{1}\left(T_{1}\right) \frac{\partial T_{1}}{\partial x}=\rho L_{v} V_{1}-Q\left(t, T_{1}\right), V_{1}=V_{*} \sqrt{\frac{T_{v}}{T_{1}}} \exp \left(-\frac{T_{*}}{T_{1}}\right), \\
& \quad x=s_{2}(t): \lambda_{2}\left(T_{m}\right) \frac{\partial T_{2}}{\partial x}=\lambda_{1}\left(T_{m}\right) \frac{\partial T_{1}}{\partial x}+\rho L_{m} V_{2}, T_{1}=T_{2}=T_{m}, \\
& \quad x=+\infty: T_{2}=T_{\infty},
\end{aligned}
$$


where $T_{v}, T_{m}, T_{\infty}$ are the known temperatures of evaporation (under normal atmospheric pressure), melting and solid phase of metal, respectively; $\lambda_{k}\left(T_{k}\right), c_{k}\left(T_{k}\right), \rho, L_{v}$ and $L_{m}$ are the specific heat coefficients (functions), which are typical for the given metal (note, that we consider the model with constant and equal densities of solid and liquid phases of metal, i.e., $\left.\rho_{1}=\rho_{2}=\rho\right) ; s_{k}(t)$ are the phase division boundary coordinates to be found; $V_{k}(t, x)=\frac{d s_{k}}{d t}$ are the phase division boundary velocities; $T_{k}(t, x)$ are the unknown temperature fields; index $k=1,2$ corresponds to the liquid and solid phases, respectively.

We will consider the processes of melting and evaporation under a long time powerful pulse, i.e. $\sim 10^{-3} \mathrm{sec}$ and more. In this case, the function $Q\left(t, T_{1}\right)$ determining the amount of the absorbed energy is defined by the formula

$$
Q\left(t, T_{1}\right)=\chi\left(T_{1}\right) \cdot q_{0}(t)
$$

where $q_{0}(t)$ is the power of laser pulse, assumed to be a constant $q_{0}$, and $\chi\left(T_{1}\right)$ is the absorbtion coefficient of the energy. Velocity $V_{*}$, which is approximately equal velocity of sound in the metal, can be given by the formula [39]

$$
V_{*}=\frac{P_{a} \sqrt{A}}{\rho \sqrt{2 \pi R T_{v}}} \exp \frac{T_{*}}{T_{v}}, \quad T_{*}=\frac{A L_{v}}{R},
$$

where $P_{a}$ is the normal atmospheric pressure, $A$ is the atomic weight, $R$ is the universal gas constant.

Since our aim is to demonstrate that the formulae obtained above produce realistic data, we need to specify all coefficients arising in BVP (89)-(93). Thus, all coefficients were taken from the paper [39], where the processes of melting and evaporation of aluminium were studied. In the particular case, the temperature dependence of the quantities $c_{k}\left(T_{k}\right)$ and $\chi\left(T_{1}\right)$ are essential and have the form

$$
\begin{gathered}
c_{1}\left(T_{1}\right)=1086, T \geq T_{m}, \quad c_{2}\left(T_{2}\right)=752.2+0.473 \cdot T, T<T_{m}, \\
\chi\left(T_{1}\right)=0.64\left(\frac{T_{1}}{11600}\right)^{0.4},
\end{gathered}
$$

where $\left[c_{k}\left(T_{k}\right)\right]=\mathrm{Jkg}^{-1} \mathrm{~K}^{-1}$. Other physical quantities are assumed to be some constants, namely: $\lambda_{1}=\lambda_{2}=240 \mathrm{Wm}^{-1} \mathrm{~K}^{-1}, \rho=2545 \mathrm{kgm}^{-3}, L_{m}=0.64 \cdot 10^{6} \mathrm{Jkg}^{-1}, L_{v}=10.8 \cdot 10^{6} \mathrm{Jkg}^{-1}$, $T_{v}=2793 \mathrm{~K}, T_{m}=933 \mathrm{~K}, T_{\infty}=300 \mathrm{~K}$.

First of all, to use the results obtained above, we should transform the governing equations of BVP (⒐ $-(\underline{93})$ to the form (28) and (29). Using Goodman's substitution

$$
u=\phi_{1}\left(T_{1}\right) \equiv \int_{0}^{T_{1}} c_{1}(\zeta) \rho d \zeta, \quad v=\phi_{2}\left(T_{2}\right) \equiv \int_{0}^{T_{2}} c_{2}(\xi) \rho d \xi .
$$

one easy transforms BVP (89)-(193) to the form (28)-(32), where

$$
\begin{gathered}
d_{1}(u)=\frac{\lambda_{1}}{\rho c_{1}}, \quad d_{2}(v)=\frac{\lambda_{2}}{\rho} \cdot \frac{1}{\sqrt{a_{2}^{2}+2 \frac{b_{2}}{\rho} v}}, \\
q(u)=\frac{0.64}{11600^{0.4}} \cdot \frac{q_{0}}{\left(\rho c_{1}\right)^{0.4}} u^{0.4}, \quad h(u)=\frac{P_{a} \sqrt{c_{1} A}}{\sqrt{2 \pi R \rho}} \exp \frac{T_{*}}{T_{v}} \cdot \frac{1}{\sqrt{u}} \exp \left(-\frac{\rho c_{1} T_{*}}{u}\right),
\end{gathered}
$$




$$
u_{m}=\rho c_{1} T_{m}, \quad v_{m}=\rho\left(a_{2} T_{m}+\frac{b_{2}}{2} T_{m}^{2}\right), \quad v_{\infty}=\rho\left(a_{2} T_{\infty}+\frac{b_{2}}{2} T_{\infty}^{2}\right)
$$

and

$$
H_{1}=\rho L_{v}, \quad H_{2}=\rho L_{m}
$$

In formulae (99)-(102), we use the notation $c_{2}\left(T_{2}\right)=a_{2}+b_{2} T_{2}$, where the coefficients $a_{2}$ and $b_{2}$ are determined by (96).

Now, using formulae (82) and (83), and (87) and (88), and making the relevant simplifications, one can receive the exact solution of BVP (89)-(93) in the explicit form

$$
\begin{gathered}
T_{1}=T_{m}+\frac{\left(K+h^{-1}(\mu)-\rho c_{1} T_{m}\right) e^{-\frac{\mu \rho c_{1}}{\lambda_{1}} \xi}-K}{\rho c_{1}} \\
T_{2}=\frac{T_{\infty} c_{2}\left(\frac{T_{m}+T_{\infty}}{2}\right)+\left(T_{m}-T_{\infty}\right) c_{2}\left(\frac{T_{\infty}}{2}\right) e^{-\frac{\mu \rho c_{2}\left(T_{\infty}\right)}{\lambda_{2}}(\xi-\delta)}}{c_{2}\left(\frac{T_{m}+T_{\infty}}{2}\right)-\frac{b_{2}}{2}\left(T_{m}-T_{\infty}\right) e^{-\frac{\mu \rho c_{2}\left(T_{\infty}\right)}{\lambda_{2}}(\xi-\delta)}} \\
\delta=\frac{\lambda_{1}}{\mu \rho c_{1}} \log \left(1+\frac{h^{-1}(\mu)-\rho c_{1} T_{m}}{K}\right),
\end{gathered}
$$

where $K=\left(T_{m}-T_{\infty}\right) \rho c_{2}\left(\frac{T_{m}+T_{\infty}}{2}\right)+H_{2}$ and the velocity $\mu$ satisfies the transcendent equation

$$
\frac{q\left(h^{-1}(\mu)\right)}{\mu}-h^{-1}(\mu)=K-\rho c_{1} T_{m}+H_{1} .
$$

Equation (106) can be easy solved numerically by means of Maple (Mathematica etc.) program package. We used Maple 12. The calculations were carried out for two values of the parameter $q_{0}$ : 1) $q_{0}=1 \cdot 10^{10} \mathrm{Wm}^{-2}$; 2) $q_{0}=5 \cdot 10^{10} \mathrm{Wm}^{-2}$. The following phase division boundary velocities $\left([\mu]=\mathrm{m} \cdot \mathrm{c}^{-1}\right)$ were obtained:

$$
\mu=\left\{\begin{array}{l}
0.10 \text { if } q_{0}=1 \cdot 10^{10} \\
0.54 \text { if } q_{0}=5 \cdot 10^{10}
\end{array}\right.
$$

The corespondent liquid phase thickness $([\delta]=\mathrm{m})$ is

$$
s_{2}(t)-s_{1}(t)=\delta=\left\{\begin{array}{lll}
9.60 \cdot 10^{-4} & \text { if } & q_{0}=1 \cdot 10^{10} \\
2.23 \cdot 10^{-4} & \text { if } & q_{0}=5 \cdot 10^{10}
\end{array}\right.
$$

The temperature fields of liquid and solid phases of aluminium are presented in Fig.1. Comparing the temperature fields and the liquid phase thickness obtained here with those from [39], one concludes that they are sufficiently similar. Of course, one should take into account that a chain of laser pulses was used in [39] for numerical simulations, while one only laser pulse was used to obtain formulae presented above.

\section{Conclusions}

In this paper, nonlinear boundary value problems by means of the classical Lie symmetry method are studied. First of all, an analysis of the known definitions of Lie invariance for BVPs is presented. Having this done, a new definition of invariance in Lie sense for BVPs of the form (4)-(7) is formulated. This definition (see Definition 2) is applicable to a wide class of BVPs, including those with several basic equations, with moving boundaries, and with 

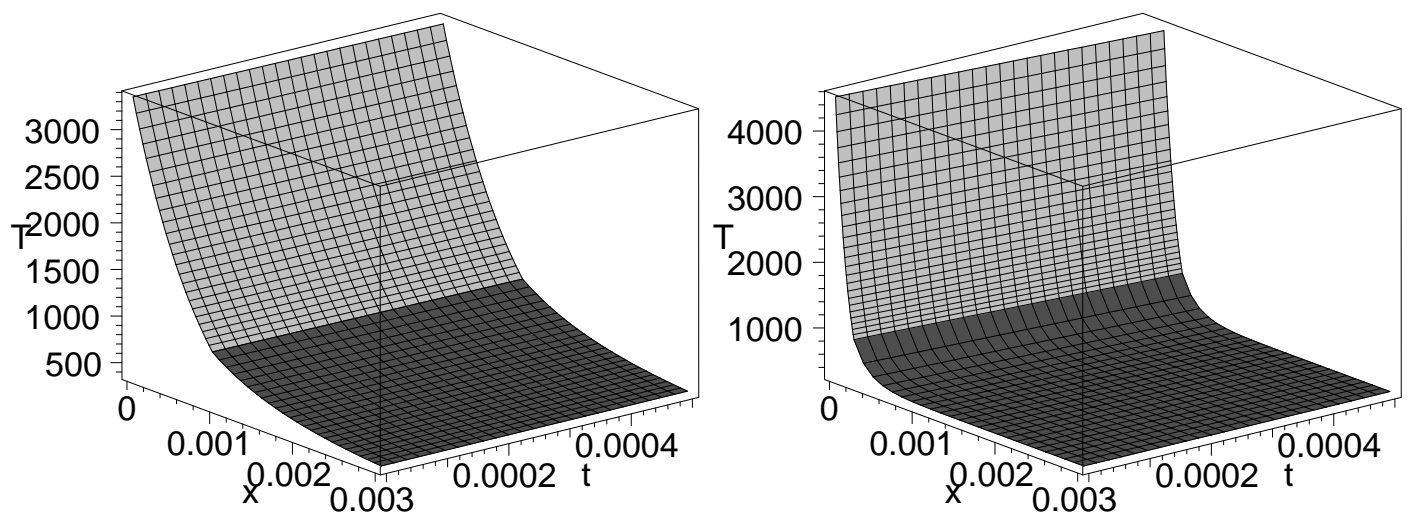

Figure 1: Exact solutions of problem (89)-(93) for aluminium with the energy flux: 1) $q_{0}=$ $1.0 \cdot 10^{10} \mathrm{Wm}^{-2}$ and 2) $q_{0}=5.0 \cdot 10^{10} \mathrm{Wm}^{-2}$.

boundary conditions on non-regular manifolds, moreover, it can be easily generalized on BVPs with hyperbolic and elliptic basic equations.

The class of two-dimensional nonlinear BVPs (28)-(32), modeling the process of melting and evaporation of metals (under acting a powerful flux of energy) is studied in details. Using Definition 2, all possible Lie symmetries (see Theorem 3) and the relevant reductions with physical meaning to BVPs for ordinary differential equations (see Theorems 4 and 5) are constructed. The example how to construct exact solution of the nonlinear problem (28)-(32) with correctly-specified coefficients for aluminium is also presented. We established that exact formulae (103)-(105), obtained by direct application of Theorem 4, lead to quite realistic results, which are sufficiently similar to those obtained earlier by numerical simulations.

The work is in progress to extend the results on multidimensional BVPs using the definition proposed in this paper.

\section{References}

[1] L.V. Ovsiannikov, The group analysis of differential equations, Academic Press, New York,1982.

[2] G.W. Bluman, S. Kumei, Symmetries and differential equations, in: Applied Mathematical Science, vol. 81, Springer, Berlin,1989.

[3] P.J. Olver, Applications of Lie groups to differential equations, second ed., in: Graduate Texts in Mathematics, vol. 107, Springer, New York, 1993.

[4] W.I. Fushchych, W.M. Shtelen, M.I. Serov, Symmetry analysis and exact solutions of equations of nonlinear mathematical physics, Kluwer, Dordrecht, 1993.

[5] G.W. Bluman, S.C. Anco, Symmetry and integration methods for differential equations, in: Applied Mathematical Science, vol. 154, Springer, New York, 2002.

[6] N.H. Ibragimov, Transformation groups applied to mathematical physics, D. Reidel Publishing Co., Dordrecht, 1985. 
[7] A.F. Sidorov, V.P. Shapeev, N.N. Yanenko, The method of differential constraints and its applications in gas dynamics, Nauka, Novosibirsk, 1984 (in Russian).

[8] P.J. Olver, Direct reduction and differential constraints, Proc. Roy. Soc. Lond. A 444 (1994) 509-523.

[9] V.A. Galaktionov, S.R. Svirshchevskii, Exact solutions and invariant subspaces of nonlinear partial differential equations in mechanics and physics, Chapman \& Hall/CRC, Boca Raton, 2007.

[10] W. Malfliet, W. Hereman, The tanh method. I: Exact solutions of nonlinear evolution and wave equations, Phys. Scr. 54 (1996) 563-568.

[11] N.A. Kudryashov, Simplest equation method to look for exact solutions of nonlinear differential equations, Chaos, Solitons and Fractals, 24 (2005) 1217-1231.

[12] A.M. Wazwaz, The tanh-coth method for solitons and kink solutions for nonlinear parabolic equations, Appl. Math. Comput. 188 (2007) 1467-1475.

[13] M. Wang, X. Li, J. Zhang, The $\frac{G^{\prime}}{G}$-expansion method and evolution equations in mathematical physics, Phys. Lett. A 372 (2008) 417-423.

[14] W.X. Ma, T.W. Huang, Y. Zhang, A multiple exp-function method for nonlinear differential equations and its application, Phys. Scr. 82 (2010) 065003.

[15] R. Cherniha, A constructive method for construction of new exact solutions of nonlinear evolution equations, Rep. Math. Phys. 38 (1996) 301-312.

[16] R.M. Cherniha, New non-Lie ansätze and exact solutions of nonlinear reaction-diffusionconvection equations, J. Phys. A: Math. Gen. 31 (1998) 8179-8198.

[17] W.X. Ma, J.H. Lee, A transformed rational function method and exact solutions to the $3+1$ dimensional Jimbo-Miwa equation, Chaos, Solitons and Fractals, 42 (2009) 1356-1363.

[18] A.D. Polyanin, V.F. Zaitsev, Handbook of nonlinear differential equations, CRC Press Company, Boca Raton, 2004.

[19] V.V. Pukhnachov, Invariant solutions of the Navier-Stokes equations describing motion with a free boundary, Dokl. Akad. Nauk SSSR 202 (1972) 302-305 (in Russian).

[20] G. Bluman, Application of the general similarity solution of the heat equation to boundary value problems, Quart. Appl. Math. 31 (1974) 403-415.

[21] V.K. Andreev, O.V. Kaptsov, V.V. Pukhnachov, A.A. Rodionov, Application of grouptheoretical methods in hydrodynamics, Kluwer Academic Publishers, Netherlands, 1998.

[22] C. Rogers, W.F. Ames, Nonlinear boundary value problems in science and engineering, in: Mathematics in Science and Engineering, vol. 183, Academic Press, Boston, 1989.

[23] V. Alexiades, A.D. Solomon, Mathematical modeling of melting and freezing processes, Hemisphere Publishing Corporation, Washington, 1993.

[24] N.F. Britton, Essential mathematical biology, Springer, Berlin, 2003. 
[25] J. Crank, Free and moving boundary problems, Clarendon Press, Oxford, 1984.

[26] J. Ready, Effects of high-power laser radiation, Academic Press, New York, 1971.

[27] L.I. Rubinstein, The Stefan problem, in: Translations of Mathematical Monographs, vol. 27, Amer. Math. Soc., Providence, 1971.

[28] J. Stefan, Über einige probleme der theorie der wärmeleitung, S. B. Wien. Akad. Mat. Natur. 98 (1889) 173-184.

[29] A.C. Briozzo, D.A. Tarzia, An explicit solution for an instantaneous two-phase Stefan problem with nonlinear thermal coefficients, IMA J. Appl. Math. 67 (2002) 249-261.

[30] R.M. Cherniha, N.D. Cherniha, Exact solutions of a class of nonlinear boundary value problems with moving boundaries, J. Phys. A: Math. Gen. 26 (1993) L935-L940.

[31] R.M. Cherniha, I.G. Odnorozhenko, Exact solutions of a nonlinear boundary value problem of melting and evaporation of metals under the action of high energy flux, Dopovidi Akad. Nauk Ukrainy, ser.A 12 (1990) 44-47 (in Ukrainian, Summary in English).

[32] P. Broadbridge, B.M. Pincombe, The Stefan solidification problem with nonmonotonic nonlinear heat diffusivity, Math. Comput. Modelling 23 (1996) 87-98.

[33] V.R. Voller, J.B. Swenson, C. Paola, An analytical solution for a Stefan problem with variable latent heat, Int. J. Heat Mass Transfer 47 (2004) 5387-5390.

[34] J. Lorenzo-Trueba, V. R.Voller, Analytical and numerical solution of a generalized Stefan problem exhibiting two moving boundaries with application to ocean delta formation, J. Math. Anal. Appl. 366 (2010), 538-549.

[35] S.I. Barry, J. Caunce, Exact and numerical solutions to a Stefan problem with two moving boundaries, Appl. Math. Model. 32 (2008) 83-98.

[36] T.B. Benjamin, P.J. Olver, Hamiltonian structure, symmetries and conservation laws for water waves, J. Fluid Mech. 125 (1982) 137-185.

[37] R. Cherniha, S. Kovalenko, Exact solutions of nonlinear boundary value problems of the Stefan type, J. Phys. A: Math. and Theor. 42 (2009) 355202.

[38] V.V. Pukhnachov, Symmetry in Navier-Stokes equations, Uspekhi mechaniki 1 (2006) 6-76 (in Russian).

[39] R.M. Cherniha, I.G. Odnorozhenko, Studies of the processes of melting and evaporation of metals under the action of laser radiation pulses, Promyshlennaya Teplotekhnika 13 (1991) 51-59 (in Russian, Summary in English).

[40] J.R. King, Exact results for the nonlinear diffusion equations $\frac{\partial u}{\partial t}=\frac{\partial}{\partial x}\left(u^{-\frac{4}{3}} \frac{\partial u}{\partial x}\right)$ and $\frac{\partial u}{\partial t}=$ $\frac{\partial}{\partial x}\left(u^{-\frac{2}{3}} \frac{\partial u}{\partial x}\right)$, J. Phys. A: Math. Gen. 24 (1991) 5721-5745.

[41] H.S. Carslaw, J.C. Jager, Conduction of heat in solids, second ed., Clarendon Press, Oxford, 1959.

[42] R. Cherniha, J.R. King, Nonlinear reaction-diffusion systems with variable diffusivities: Lie symmetries, ansätze and exact solutions, J. Math. Anal. Appl. 308 (2005) 11-35. 